\title{
Impacts and Risks of Adopting Clinical Decision Support Systems
}

\author{
Wilfred Bonney \\ Capella University/ HL7 International \\ Canada
}

\section{Background}

The development of interoperable Healthcare Information Systems such as Electronic Health Record (EHR), Electronic Medical Record (EMR), and Personal Health Record (PHR) has created a platform environment whereby massive data is collected, stored, shared, and analyzed at the point of care to support patient outcomes and efficient healthcare delivery. However, the analysis of structured and unstructured clinical datasets to support the decision-making process of healthcare providers is proving to be difficult in the healthcare industry as data is being captured in different and/or multiple formats. Clinical Decision Support Systems (CDSS) have emerged as toolkits for efficiently managing and analyzing the clinical datasets stored in the repositories of EHR, EMR, and PHR. In this book chapter, a literature review is used to explore and answer the following questions:

- What are clinical decision support systems (CDSS)?

- How does CDSS influence the decision-making process of clinicians in medical practice?

- What are the significant impacts and risks associated with the use and adoption of CDSS?

\section{Introduction}

Increasing in computing power has provided a platform for developers to build numerous computing applications that "would have been impossible just a few years ago" (Seltzer, 2005, p. 50). Clinical Decision Support Systems (CDSS) are computer-based healthcare applications used to integrate clinical and patient information to provide support for decision-making in patient care as well as to generate case-specific advices (Bonney, 2009; Kotze \& Brdaroska, 2004). CDSS aid in clinical decision-making not only by providing physicians and other healthcare stakeholders with computerized advice regarding drug doses, medications, laboratory results and diagnosis but also by enhancing a clinician's ability to process data and information (Bonney, 2009; Kaushal, Shojania, \& Bates, 2003; K. Kawamoto, Houlihan, Balas, \& Lobach, 2005). However, there is growing evidence that when poorly designed, deployed and/or used, CDSS may lead to more harm than good (Coiera, Westbrook, \& Wyatt, 2006; Kotze \& Brdaroska, 2004; Toth-Pal, Wårdh, Strender, \& Nilsson, 2008).

This paper aims to explore the significant impacts and risks of adopting CDSS in clinical practice. Whereas the impact factors will explore how the use of CDSS has impacted clinical 
decision-making, clinical practice guidelines, efficiency of healthcare delivery, and patient safety and outcomes; the risk factors will focus on the CDSS dependence on repositories, knowledge management, misinterpretation of clinical datasets, and failure to fit routine works of clinicians.

\section{Methodology}

A literature review is used to highlight the relevant impacts and risks of adopting CDSS in clinical practice. The methodology involves a systematic review of relevant publications, found and accessed with the help of ProQuest (with multiple databases option) and EBSCOhost databases. Additional sources were retrieved using the ScienceDirect, PubMed and ACM digital libraries. Whereas the impact factors explore how the use of CDSS has impacted clinical decision-making, clinical practice guidelines, efficiency of healthcare delivery, and patient outcomes and safety; the risk factors focus on the CDSS dependence on repositories, knowledge management, misinterpretation of clinical datasets, and failure to fit routine works of clinicians.

\section{Overview of Clinical Decision Support Systems (CDSS)}

CDSS have been recognized as promising tools for influencing healthcare provider performance to improve and streamline the quality of healthcare delivery (Bassa et al., 2005; Pearson et al., 2009). CDSS originated from Decision Support Systems (DSS). According to Donzelli (2006), DSS simply “combine individuals' and computers' capabilities to improve the quality of decisions" (p. 67). These functionalities and capabilities of DSS have contributed to its popularity and use in the healthcare domain. Hwang, Chang, Hung, Sung, and Yen (2004) asserted that a "DSS that supports physicians with the potential to minimize practice variation and improve patient care" (p. 240) is known as CDSS.

Throughout their inception in the medical arena in the early 1970s, CDSS have evolved immensely to support the workflow of clinicians and improved the effectiveness of decision outcomes (Bassa et al., 2005; Hwang et al., 2004; Pearson et al., 2009). Although several challenges are facing the use and adoption of CDSS in the healthcare setting, the technology still remains promising when it comes to its ability to support evidence-based practice and enhancing the clinical decision-making process of healthcare providers. It is in this regard that Kawamoto et al. (2005) noted that CDSS provide "clinicians with patient-specific assessments or recommendations to aid clinical decision making" (p. 765). Examples of CDSS include technologies such as Computerized Physician Order Entry (CPOE) systems that provide patient-specific recommendations as part of the order entry process; outpatient systems that attach care reminders to the charts of patients in need of specific preventive care services; and laboratory alerting systems that page physicians when critical laboratory values are detected (Kawamoto \& Esler, 2006).

The architecture components of CDSS consist of knowledge base, inference/reasoning engine, and user communication/interaction (Kola, n.d.; O'Kane et al., 2010). Figure 1 shows the architecture components of CDSS. Whereas the knowledge base is made up guidelines, rules, and probabilistic models, the inference/reasoning engine combines the data in the knowledge base with that of the patient data. The user communication component of the architecture consists of a simple way of getting data into the system and getting results to the user (O'Kane et al., 2010; Berner \& La Lande, 2007). The fact that the architecture of the 
CDSS depends on knowledge bases means that inappropriate representation of data, information, and knowledge present enormous threats to the adoption of CDSS in clinical practice.

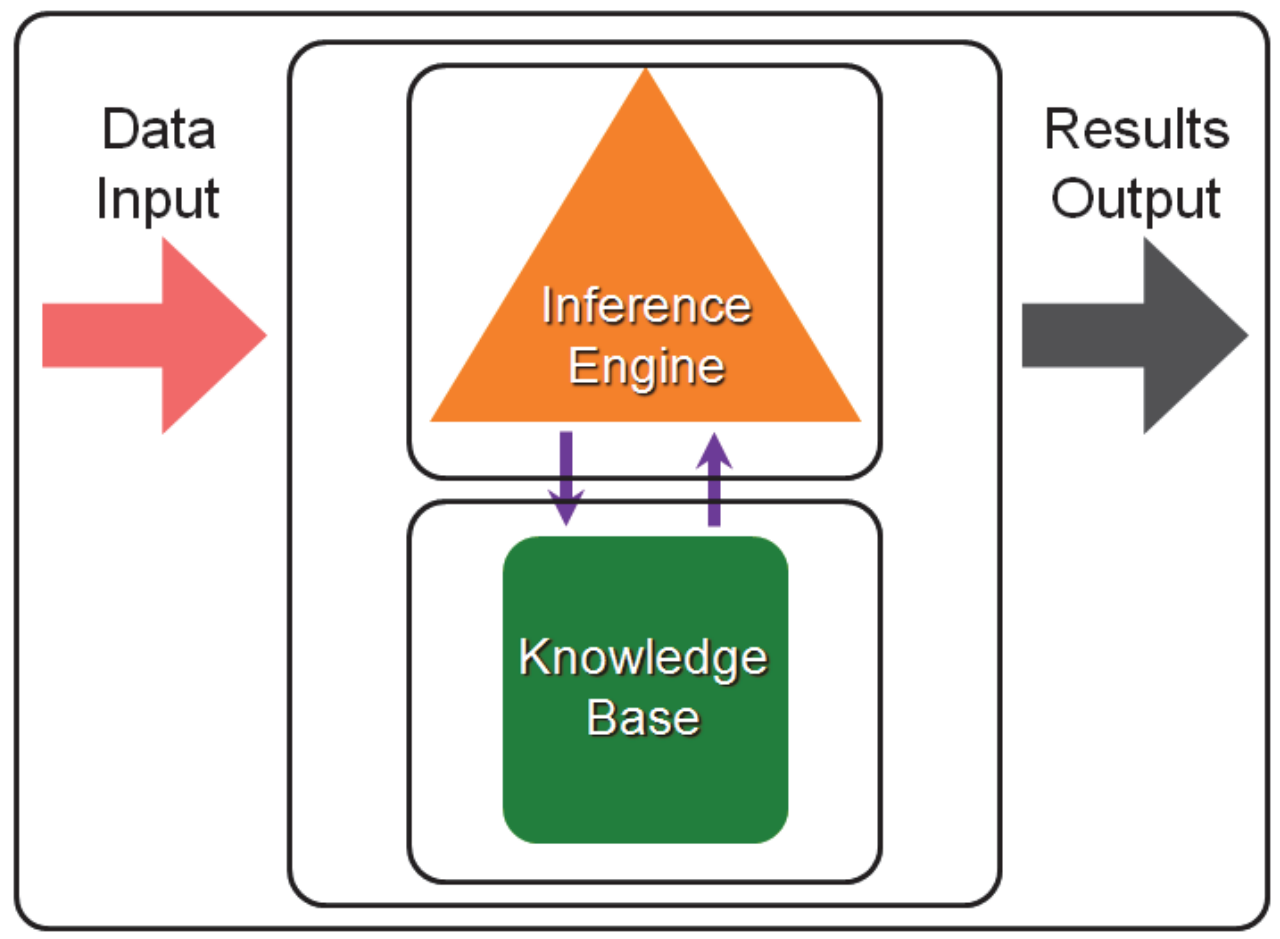

Fig. 1. Architecture components of CDSS (Kola, n.d.)

\section{Impact factors}

The impact factors associated with the use and adoption of CDSS could be categorized under five broad themes: clinical decision-making, clinical practice guidelines, efficiency of healthcare delivery, and patient safety and outcomes.

\subsection{Clinical decision-making}

CDSS has a significant impact on the quality of decision making by healthcare providers. According to Kawamoto et al. (2005), CDSS provide "clinicians with patient-specific assessments or recommendations to aid clinical decision making" (p. 765). However, this goal of achieving quality decision making is not an easy endeavour. Clinical decisionmaking is a "complex task requiring a knowledgeable practitioner, reliable informational inputs, and a supportive environment" (O'Neill, Dluhy, \& Chin, 2005, p. 69). According to Buckingham (2002), clinical decision-making consists of classification tasks "where cues are used to assign patients to one of a number of potential categories" (p. 238). This complexity of achieving quality clinical decision making by healthcare providers is often facilitated with the use of CDSS as a supportive tool. 
In an attempt to improve the use of CDSS to support quality decision making in clinical practice, Buckingham (2002) proposed a gelatean model with the goal of linking "intuitive explanations of clinical expertise with empirical data analysis to enhance judgement accuracy" (p. 250). Buckingham (2002) identified this relation as a symbiotic relationship between clinicians and computers. Whereas the clinicians are responsible for using their psychological validity, the computers' side of the symbiosis comes with its powers of data storage and analysis (Buckingham, 2002, p. 249). Enhancing judgement accuracy of clinicians is critical in ensuring that information emanating from the CDSS are interpreted well by the attending clinicians and not misinterpreted. Physicians can enhance their clinical judgement accuracy by combining their experiential knowledge with the use of CDSS so that a symbiotic relationship can be established.

\subsection{Clinical practice guideline}

Many healthcare providers depend on clinical practice guidelines for quality and evidencebased healthcare delivery. Clinical practice guidelines are "systematically developed statements to assist practitioners and patient decisions about appropriate health care for specific clinical circumstances" (Kotze \& Brdaroska, 2004, p. 361). According to Kotze and Brdaroska (2004), clinical practice guidelines have "little influence upon clinician practice and patient outcomes unless they are effectively implemented and integrated into the clinical setting" (p. 362).

One approach for effectively integrating clinical practice guidelines into medical practice is the use of CDSS. The use of CDSS has facilitated clinicians' adherence to clinical practice guidelines, thereby improving patient outcomes (Kotze \& Brdaroska, 2004; Kwok, Dinh, Dinh, \& Chu, 2009). Kotze and Brdaroska (2004) noted that the "ability of computers to store, search and sort large volumes of data rapidly, as well as the everexpanding knowledge, access and use of computers, have paved the way for the incorporation of clinical practice guidelines into computer-based decision support systems" (p. 362). This is because not only does the use of CDSS demand clinical practice guidelines but it also makes it easier for programmers to develop rule-based and/or case-based reasoning to support the advices emanating from the CDSS.

The encoded rules in the clinical practice guidelines provide the framework in which programming rules are encoded and used in the development of CDSS. For example, Kwok et al. (2009) found that the use of an integrated and dynamic electronic decision support system (EDSS) at a single emergency department promoted strict adherence to asthma clinical guidelines and improved clinical documentation and discharge management plans for asthma management. It is in this regard that Kotze and Brdaroska (2004) indicated that CDSS are "crucial elements in long-term strategies for promoting the use of clinical practice guidelines" (p. 362).

\subsection{Efficiency of healthcare delivery}

In a study conducted to assess the impact of CDSS on the management of patients with Hypercholesterolemia, Bassa et al. (2005) found that "it is possible to optimize the efficiency of the management of hypercholesterolemia in standard practice by the implementation of a CDSS" (p. 71). In a similar study, Cobos et al. (2005) found that the use and adoption of CDSS in clinical practice "was as effective as usual care and induced important savings in the management of hypercholesterolemia" (p. 431). The above two studies contribute to our 
understanding about the need to implement CDSS in clinical practice so as to support the efficiency of healthcare delivery.

Healthcare providers stand to gain enormously and streamline the workflow of physicians by adopting CDSS. For example, Pomerleau (2008) noted that the use of CDSS allow "nurses to have information and unit policies at their fingertips, which help them adhere to standards while at the bedside" (p. 154). Successful implementation of CDSS in clinical settings will reduce waiting times, minimize the length of stay in hospitals, and enhance the efficiency of healthcare delivery.

\subsection{Patient safety and outcomes}

Improving patient outcomes requires the use of efficient decision-making process and evidence-based practice that can only be best achieved through the utilization of CDSS. One of the ultimate uses of CDSS is to improve patient safety and outcomes. CDSS have consistently shown great promise for reducing medical errors and improving patient care, safety, and outcomes (K. Kawamoto et al., 2005; Mahoney, Berard-Collins, Coleman, Amaral, \& Cotter, 2007; Pomerleau, 2008; Sintchenko, Coiera, Iredell, \& Gilbert, 2004; Subramanian et al., 2007). When it comes to the use of medications and diagnostic testing in clinical settings, CDSS has emerged as a technology to reduce medication errors, "improve diagnostic accuracy, provide easier and more rapid access to patient information and more complete medical records" (Courtney, Alexander, \& Demiris, 2008, p. 692).

According to Mahoney et al. (2007), medication errors are deleterious, prevalence and costly. Hence the need to use robust healthcare information systems to monitor, track, and manage medications administered to patients is of prime concern to many healthcare providers. Mahoney et al. (2007) found that the use of integrated clinical information system technology "decreased selected types of medication errors throughout the medication-use process in a health care system and improved therapeutic drug monitoring in patients" (p. 1969). In the context of identifying the potential adverse drug events (ADEs) at the medication ordering stage, Roberts et al. (2010) noted that successful implementation of CPOE and other advanced CDSS tools "significantly increased the number of potential ADE alerts for pharmacist review and the number of true-positive ADE alerts identified per 1000 admissions" (p. 1845).

Moreover, in a randomized control trial conducted to evaluate the effectiveness of CDSS in reducing potentially inappropriate prescribing to older adults, Terrell et al. (2009) found that CPOE with decision support "significantly reduced prescribing of potentially inappropriate medications for seniors" (p. 1389). In another study, Subramanian et al. (2007) found that the increasing use of CPOE has facilitated the "elimination of handwriting identification problems, reductions in error associated with similar drug names, faster delivery of orders to the pharmacy" (p. 1451). These studies and others from the literature affirm the significant impact of CDSS in reducing medical errors in clinical practice, thereby, improving the quality of care, patient safety, and patient outcomes (Pearson et al., 2009).

\section{Risk factors}

The risk factors focus on the CDSS dependence on repositories, knowledge management, misinterpretation of clinical datasets, and failure to fit routine works of clinicians. 


\subsection{Dependence on repositories}

One of the critical architecture components of all CDSS is the knowledge base. The knowledge base depends on a centralized clinical data repository (K. Kawamoto, Lobach, Willard, \& Ginsburg, 2009; Roberts et al., 2010). The fact that CDSS depends on good quality clinical data repository reinforces the need for standardized data representation, storage, and retrieval that can be centrally managed in the knowledge base repositories. Lack of good clinical data warehouse could have significant impact on the quality of advices emanating from CDSS. Data mining algorithms require good quality clinical data repositories to be able to extract knowledge to support clinical decision-making.

CDSS also depend profoundly on large volumes of readily-accessible, existing clinical datasets (Bonney, 2009). These large volumes of data are usually extracted from the repository content of EHR, EMR and PHR. Lack of standardized data capture by these systems will lead to corrupt datasets. When the entries in these data repository are not coded appropriately, there is tendency that the resulting datasets will not be a good representative of the patient population (Bonney, 2009). It is therefore essential that standardized data representation are used for leveraging the knowledge base repositories contained in the CDSS so as to facilitate the generation of patient-specific care recommendations at the point of care (K. Kawamoto et al., 2009).

\subsection{Knowledge management}

CDSS depend on appropriate implementation of knowledge management. According to Kalkan (2008), the whole concepts of data, information and knowledge are generally misunderstood. Acknowledging the fact that information results from replacing data within some meaningful content, Kalkan (2008) noted that knowledge is an "organized and transformed combination of information, assimilated with a set of rules, procedures and operations learnt through experience and practice" (p. 391). This definition of knowledge emphasizes the need to manage knowledge appropriately. Without proper set of rules, guidelines and operations, knowledge cannot be assimilated. Thus the need for knowledge management in CDSS cannot be ignored.

Knowledge management is defined as a "systematic management of knowledge-related activities, practices, programs and policies within the enterprise" (Kalkan, 2008, p. 392). Knowledge management has gain popularity in the IT industry because of its emphasis on how to articulate, capture and distribute explicit and tacit knowledge in different formats (Herschel \& Jones, 2005; Kalkan, 2008). Knowledge management activities aim to "effectively apply an organization's knowledge to create new knowledge to achieve and maintain competitive advantage" (Kalkan, 2008, p. 392). Creating new knowledge in the medical field is crucial in helping healthcare providers in combating new diseases and symptoms. However, when the newly created knowledge is based on poor quality data, the resulting outcome could be very devastating in clinical settings.

The fact that CDSS have "become increasingly sophisticated by matching patient characteristics with computerised knowledge bases and using algorithms to generate patient-specific assessments or treatment recommendations" (Pearson et al., 2009, p. 155) demand that appropriate management of knowledge is implemented in the CDSS to ensure that the patient-specific assessments and/or treatment recommendations are not based on poor quality data. It is therefore important that narrative information emanating from the CDSS is further processed and analyzed by healthcare providers before clinical decisions are made (Pearson et al., 2009). 


\subsection{Misinterpretation of clinical datasets}

Clinical information stored in the CDSS are often misrepresented and misinterpreted. This is partly due to the inconsistencies in data coding and extraction of poor quality data. According to Coiera et al. (2006), the use of CDSS can "improve the overall safety and quality of health care delivery, but may also introduce machine-related errors" (p. 20). Coiera et al. (2006) noted that the use of poor quality data could lead to wrong medications and misdiagnosis. Coiera et al. (2006) also noted that automation biases and using evidenceretrieval systems may generate decision errors that might not necessarily correlates with the experiential knowledge of the physicians.

Acknowledging the fact that inference rules forms the basic building blocks of any given CDSS and are usually extracted by data mining existing clinical datasets, Bonney (2009) noted that the "trustworthy of CDSS is based on how effective the extracted inference rules correlates with the experiential knowledge of domain experts" (p. 116). Chaudhry (2008) also emphasized the misrepresentation of clinical datasets by noting that, "real clinical data from patient interviews or medical records are far less structured and would likely alter the performance of the system considerably" (p. 86), if not extracted appropriately. This has a significant effect on the quality of data used in developing CDSS. Poor quality data will lead to misinterpretation of clinical datasets. The use of health information standards such as ICD-10, SNOMED, LOINC and UMLS will ensure uniformity and consistency of the health datasets, used in generating the inference rules (Bonney, 2009).

\subsection{Failure to fit routine works of clinicians}

According to Hwang et al. (2004), accessing CDSS in a computer by medical practitioners is not a smooth process for actual usage/implementation. Hwang et al. (2004) attributed the complexity of the process to the fact that in actual clinical settings, integrating CDSS with the routine work of clinicians will demand that the physicians "run back and forth from point of care to computer station to complete their diagnosis" (p. 240). This approach could be daunting considering the workload of average physicians. Moreover, the routine use of CDSS during consultation could alienate patients from the direct contact with their physicians.

When it comes to the use and adoption of technology, medical practitioners with experiential knowledge are more likely to override the decisions and advices presented by CDSS. For example, Dowding et al. (2009) noted that nurses are "less likely to use CDSS for telephone triage decisions that they feel they have experience in making" (p. 1160). These attitudes of medical practitioners towards CDSS often impede their overall acceptance and adoption in clinical practice.

Acknowledging the fact that perceived usefulness of medical information is a function of its relevance, validity, and the effort involved in searching for it, Sintchenko et al. (2004) noted that physicians often "choose not to use available evidence at the time of decision making but rely on what they know and choose the strategy requiring least effort" (p. 75). Hence clinicians' attitudes and the environment in which decisions are made influence the overall acceptance and adoption of decision support tools (Sintchenko et al., 2004; Toth-Pal et al., 2008). It is therefore recommended that the development and deployment of the CDSS should fit the workflow of clinicians so as to ensure that the system is enabling without constraining (Ash, Gorman, Lavelle, \& Payne, 2003; Bonney, 2009). 


\section{Discussion and conclusion}

In a qualitative study conducted to explore general practitioners' (GPs) handling of a CDSS during the implementation process, Toth-Pal et al. (2008) found that despite their benefits in medicine, CDSS are rarely used in clinical practice. Toth-Pal et al. (2008) attributed CDSS barriers to "limited computer skills, shortage of time during consultation, problems with interpreting the recommendations given, and the GPs' concerns about patient reactions" (p. 40).

Moreover, in an analysis of 70 randomized controlled trial, Kawamoto et al. (2005) found that successful implementation of CDSS should "(a) provide decision support automatically as part of clinician workflow, $(b)$ deliver decision support at the time and location of decision making, (c) provide actionable recommendations, and (d) use a computer to generate the decision support" (p. 771). These four recommendations seem to support the overall use of CDSS in improving the quality of clinical care. They also make it easier for clinicians to use CDSS thereby minimising the effort required by clinicians to receive and act on system recommendations (Kawamoto et al., 2005). The development of CDSS should also utilize health information standards so as to ensure its interoperability with other legacy systems and support distributed computing (Bonney, 2009).

This research has the potential to benefit healthcare providers and stakeholders in determining the significant impacts and risks of adopting CDSS in medical practice. With the impacts and risks presented in the paper, it is evident that the appropriate use CDSS with emerging technologies could enhance the adoption and acceptance rate of CDSS in clinical practice. Future research should therefore focus on how to integrate Business Intelligence (BI) into CDSS. This is because BI is emerging as the new frontier in data mining that will facilitate the extraction of both structured and unstructured datasets. It is also important that future research promote the rigorous testing of CDSS to provide high quality evidence about their clinical and economic impacts on healthcare delivery (Pearson et al., 2009).

\section{References}

Ash, J. S., Gorman, P. N., Lavelle, M., \& Payne, T. H. (2003). A cross-site qualitative study of physician order entry. Journal of the American Medical Informatics Association, 10(2), 188-200.

Bassa, A., Miguel, d. V., Cobos, A., Torremadé, E., Bergoñón, S., Crespo, C., Espinosa, C. (2005). Impact of a clinical decision support system on the management of patients with hypercholesterolemia in the primary healthcare setting. Disease Management $\mathcal{E}$ Health Outcomes, 13(1), 65-72.

Berner, E. S., \& La Lande, T. J. (2007). Overview of clinical decision support systems. In Clinical decision support systems - theory and practice, (2nd ed.)., Berner E.S (ed.), Springer: New York.

Bonney, W. (2009). Is it appropriate, or ethical, to use health data collected for the purpose of direct patient care to develop computerized predictive decision support tools? Studies in Health Technology and Informatics, 143, 115-121.

Buckingham, C. (2002). Psychological cue use and implications for a clinical decision support system. Medical Informatics $\mathcal{E}$ the Internet in Medicine, 27(4), 237-251. 
Chaudhry, B. (2008). Computerized clinical decision support: Will it transform healthcare? doi:10.1007/s11606-007-0432-9

Cobos, A., Vilaseca, J., Asenjo, C., Pedro-Botet, J., Sáonchez, E., Val, A., Bergoñón, S. (2005). Cost effectiveness of a clinical decision support system based on the recommendations of the european society of cardiology and other societies for the management of hypercholesterolemia: Report of a cluster-randomized trial. Disease Management \& Health Outcomes, 13(6), 421-432.

Coiera, E., Westbrook, J. I., \& Wyatt, J. C. (2006). The safety and quality of decision support systems. Methods of Information in Medicine, 45(Suppl. 1), 20-25.

Courtney, K. L., Alexander, G. L., \& Demiris, G. (2008). Information technology from novice to expert: Implementation implications. Journal of Nursing Management, 16(6), 692699.

Donzelli, P. (2006). A decision support system for software project management. IEEE Software, 23(4), 67-75.

Dowding, D., Mitchell, N., Randell, R., Foster, R., Lattimer, V., \& Thompson, C. (2009). Nurses' use of computerised clinical decision support systems: A case site analysis. Journal of Clinical Nursing, 18(8), 1159-1167.

Herschel, R. T., \& Jones, N. E. (2005). Knowledge management and business intelligence: The importance of integration. Journal of Knowledge Management, 9(4), 45-55.

Hwang, H., Chang, I., Hung, W., Sung, M., \& Yen, D. (2004). The design and evaluation of clinical decision support systems in the area of pharmacokinetics. Medical Informatics \& the Internet in Medicine, 29(3), 239-251. doi:10.1080/14639230400009158

Kalkan, V. D. (2008). An overall view of knowledge management challenges for global business. Business Process Management Journal, 14(3), 390-400.

Kaushal, R., Shojania, K. G., \& Bates, D. W. (2003). Effects of computerized physician order entry and clinical decision support systems on medication safety: A systematic review. Archives of Internal Medicine, 163(12), 1409-1416.

Kawamoto, K., \& Esler, B. (2006). HL7 service functional model specification - decision support services (DSS). Retrieved December 2, 2005, from http://www.hl7.org/documentcenter/ballots/2006SEP/support/AUDIT_SDO_C DS_DSS_R1_D1_2006SEP_20070129061919.pdf

Kawamoto, K., Houlihan, C. A., Balas, E. A., \& Lobach, D. F. (2005). Improving clinical practice using clinical decision support systems: A systematic review of trials to identify features critical to success. British Medical Journal, 330(7494), 765-772.

Kawamoto, K., Lobach, D. F., Willard, H. F., \& Ginsburg, G. S. (2009). A national clinical decision support infrastructure to enable the widespread and consistent practice of genomic and personalized medicine. BMC Medical Informatics \& Decision Making, 9(1), 17-30. doi:10.1186/1472-6947-9-17

Kola, J. (n.d.). Clinical decision support systems: Approach and use. Retrieved November, 21, 2010, from https://www.nibhi.org.uk/Health\%20Informatics/Decision\%20Support\%20Syste ms\%20Export.ppt

Kotze, B., \& Brdaroska, B. (2004). Clinical decision support systems in psychiatry in the information age. Australasian Psychiatry, 12(4), 361-364. doi:10.1111/j.14401665.2004.02127.x 
Kwok, R., Dinh, M., Dinh, D., \& Chu, M. (2009). Improving adherence to asthma clinical guidelines and discharge documentation from emergency departments: Implementation of a dynamic and integrated electronic decision support system. Emergency Medicine Australasia, 21(1), 31-37.

Mahoney, C. D., Berard-Collins, C., Coleman, R., Amaral, J. F., \& Cotter, C. M. (2007). Effects of an integrated clinical information system on medication safety in a multihospital setting. American Journal of Health-System Pharmacy, 64(18), 1969-1977.

O'Kane, T., O'Donoghue, J., Gallagher, J., Aftab, A., Casey, A., Angove, P., Courtney, G. (2010). MEWS to e-MEWS: From a paper-based to an electronic clinical decision support system. Proceedings of the European Conference on Information Management $\mathcal{E}$ Evaluation, , 301-311.

O'Neill, E., Dluhy, N. M., \& Chin, E. (2005). Modelling novice clinical reasoning for a computerized decision support system. Journal of Advanced Nursing, 49(1), 68-77.

Pearson, S., Moxey, A., Robertson, J., Hains, I., Williamson, M., Reeve, J., \& Newby, D. (2009). Do computerised clinical decision support systems for prescribing change practice? A systematic review of the literature (1990-2007). BMC Health Services Research, 9, 154-167.

Pomerleau, M. (2008). Electronic health record: Are you ready for the next step? Nursing for Women's Health, 12(2), 151-156.

Roberts, L. L., Ward, M. M., Brokel, J. M., Wakefield, D. S., Crandall, D. K., \& Conlon, P. (2010). Impact of health information technology on detection of potential adverse drug events at the ordering stage. American Journal of Health-System Pharmacy, 67(21), 1838-1846. doi:10.2146/ajhp090637

Seltzer, M. (2005). Beyond relational databases. Queue, 3(3), 50-58.

Sintchenko, V., Coiera, E., Iredell, J. R., \& Gilbert, G. L. (2004). Comparative impact of guidelines, clinical data, and decision support on prescribing decisions: An interactive web experiment with simulated cases. Journal of the American Medical Informatics Association, 11(1), 71-77.

Subramanian, S., Hoover, S., Gilman, B., Field, T. S., Mutter, R., \& Gurwitz, J. H. (2007). Computerized physician order entry with clinical decision support in long-term care facilities: Costs and benefits to stakeholders. Journal of the American Geriatrics Society, 55(9), 1451-1457.

Terrell, K. M., Perkins, A. J., Dexter, P. R., Hui, S. L., Callahan, C. M., \& Miller, D. K. (2009). Computerized decision support to reduce potentially inappropriate prescribing to older emergency department patients: A randomized, controlled trial. Journal of the American Geriatrics Society, 57(8), 1388-1394.

Toth-Pal, E., Wårdh, I., Strender, L., \& Nilsson, G. (2008). Implementing a clinical decisionsupport system in practice: A qualitative analysis of influencing attitudes and characteristics among general practitioners. Informatics for Health $\mathcal{E}$ Social Care, 33(1), 39-54. doi:10.1080/17538150801956754 


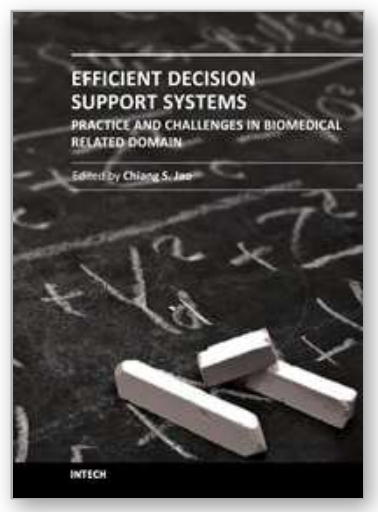

\author{
Efficient Decision Support Systems - Practice and Challenges in \\ Biomedical Related Domain \\ Edited by Prof. Chiang Jao
}

ISBN 978-953-307-258-6

Hard cover, 328 pages

Publisher InTeh

Published online 06, September, 2011

Published in print edition September, 2011

This series is directed to diverse managerial professionals who are leading the transformation of individual domains by using expert information and domain knowledge to drive decision support systems (DSSs). The series offers a broad range of subjects addressed in specific areas such as health care, business management, banking, agriculture, environmental improvement, natural resource and spatial management, aviation administration, and hybrid applications of information technology aimed to interdisciplinary issues. This book series is composed of three volumes: Volume 1 consists of general concepts and methodology of DSSs; Volume 2 consists of applications of DSSs in the biomedical domain; Volume 3 consists of hybrid applications of DSSs in multidisciplinary domains. The book is shaped decision support strategies in the new infrastructure that assists the readers in full use of the creative technology to manipulate input data and to transform information into useful decisions for decision makers.

\title{
How to reference
}

In order to correctly reference this scholarly work, feel free to copy and paste the following:

Wilfred Bonney (2011). Impacts and Risks of Adopting Clinical Decision Support Systems, Efficient Decision Support Systems - Practice and Challenges in Biomedical Related Domain, Prof. Chiang Jao (Ed.), ISBN: 978953-307-258-6, InTech, Available from: http://www.intechopen.com/books/efficient-decision-support-systemspractice-and-challenges-in-biomedical-related-domain/impacts-and-risks-of-adopting-clinical-decision-supportsystems

\section{INTECH}

open science | open minds

\section{InTech Europe}

University Campus STeP Ri

Slavka Krautzeka 83/A

51000 Rijeka, Croatia

Phone: +385 (51) 770447

Fax: +385 (51) 686166

www.intechopen.com

\section{InTech China}

Unit 405, Office Block, Hotel Equatorial Shanghai

No.65, Yan An Road (West), Shanghai, 200040, China

中国上海市延安西路65号上海国际贵都大饭店办公楼405单元

Phone: +86-21-62489820

Fax: $+86-21-62489821$ 
(C) 2011 The Author(s). Licensee IntechOpen. This chapter is distributed under the terms of the Creative Commons Attribution-NonCommercialShareAlike-3.0 License, which permits use, distribution and reproduction for non-commercial purposes, provided the original is properly cited and derivative works building on this content are distributed under the same license. 